Paper

\title{
Dynamical systems design of silicon neurons using phase reduction method
}

\author{
Kazuki Nakada ${ }^{1 a)}$, Keiji Miura ${ }^{2}$, and Tetsuya Asai ${ }^{3}$ \\ ${ }^{1}$ Graduate School of Information Sciences, Hiroshima City University, \\ 4-3-1 Ozuka-Higashi, Asa-Minami-ku, Hiroshima 731-3194, Japan \\ ${ }^{2}$ Graduate School of Science and Technology, Kwansei Gakuin University, \\ 2-1 Gakuen, Sanda 669-1337, Japan \\ ${ }^{3}$ Graduate School of Information Science and Technology, Hokkaido University, \\ Kita 14, Nishi 9, Kita-ku, Sapporo 060-0814, Japan \\ a)k.nakada@ieee.org
}

Received August 22, 2015; Revised November 25, 2015; Published April 1, 2016

\begin{abstract}
In biologically-inspired, neuromorphic engineering, it is important to design silicon neurons ( $\mathrm{SiNs}$ ) with desirable functions in a systematic way. However, the conventional design methods such as phenomenological design and the conductance-based design relied on the hand-tuned parameters. Thus a more systematic design principle, that considers mathematical structures in the dynamical systems, is needed for efficiency and robustness. In this work, we present the phase response curve (PRC)-based design for SiNs as a dynamical systems design for SiNs to enhance synchronization in an ensemble of SiNs on the basis of the phase reduction theory. By analyzing various circuit models of the previous SiNs, we explore key criteria to realize transitions between two typical PRCs, Type I and Type II. As a case study, we focus on the hybrid type SiNs for tractability and demonstrate how to tune circuit parameters of a resonate-and-fire neuron (RFN) circuit to control the peak location of phase coupling functions resulting from PRCs, which matters for phase locking.
\end{abstract}

Key Words: silicon neurons (SiNs), dynamical systems design, phase reduction theory, hybrid type spiking neurons

\section{Introduction}

Neuromorphic systems have been greatly developed and steadily redefined with the progress of the VLSI technology and the computational neuroscience over the past quarter century [1-14]. For the front-end of the intelligent and smart sensors, neuromorphic sensory systems based on silicon retina [1] and silicon cochlea [4] have been designed and fabricated. The validity and performance of such systems are verified in real-world environments [5]. For robot and biomedical applications, neuromorphic motor systems based on the central pattern generator have been implemented on various silicon platforms [6-10]. Such neuromorphic systems are integrated into fast sensory-motor processing systems $[11,12]$. More recently, cognitive neuromorphic systems exploiting spike-based computation 
have been progressed for achieving higher order brain functions including working memory, saliency detection, selective attention, and perceptual decision-making. The state of the art of the cognitive neuromorphic systems is the TrueNorth cognitive computing system recently developed by IBM [13].

Silicon neurons (SiNs) $[2,14-31]$ are one of the most fundamental elements (building blocks) constituting neuromorphic systems for spike-based computation as well as synaptic circuitries. Most of the conventional design approaches for SiNs are based on two major principles. The first is referred to as the phenomenological principle which captures some of the key behaviors and functions of neurons at an abstract level, leading to simplification of circuit dynamics and a compact circuitry [3]. The second is the conductance-based principle which emulates the dynamics of ionic channels of excitable membranes to reproduce a wide variety of behaviors and functions of neurons at detailed level, and resulting dynamics and circuits are complicated $[24,25]$. Recently, in addition to these, the dynamical systems design principle has been proposed as the third one [28-31]. This principle maps mathematical structures of neuronal dynamics, such as phase plane portrait, nullcline, and potential, onto compact silicon platforms by exploiting intrinsic characteristics of silicon devices [28-31].

The dynamical system design can be characterized by the mathematical structures embedded in target circuits and devices. From this point of view, we further classify the dynamical system design approaches for nonlinear circuits and devices into three groups, the phase plane and nullcline-based design [28-38], the potential-based design [35-37], and the phase response curve (PRC)-based design [38], as shown in Table I. These different approaches provide us with a unified perspective for tuning a vector field in phase plane in SiN design.

The phase plane and nullcline-based design approach for SiNs has been firstly established as the mathematical structure-based design [28]. In this approach, the phase plane structure of reduced system equations of ionic channel dynamics with a few state variables can be mapped onto a silicon hardware platform. By tuning nullclines on the phase plane, one can control bifurcation structure of an SiN, reproducing the functional diversity of dynamical behaviors of biological neurons, such as spike-frequency adaptation, post-inhibitory-rebound, bursting, and chaos [28, 29]. This approach can improve the design of SiNs in efficiency in a systematic way. For instance, a biophysically-inspired SiN circuitry [27] can be more sophisticated and extended to reproduce both Class 1 and Class 2 neural excitability [30]. One of the advantages of the nullcline-based design approach is to allow us to reproduce diverse dynamics of biological neurons in a compact and efficient SiN circuitry than a conductance-based SiN circuitry.

The potential-based design approach for SiN has been recently established [35]. In the potential design approach, the dynamics of an SiN can be regarded as an equation of motion of a particle on an effective potential [35]. The potential can be derived from the dynamics systematically [35]. By controlling the shape of the potential, one can tune the circuit dynamics to reproduce bursting phenomena observed in nature [35]. Beyond the scope of the SiN design, the potential-based design has been applied to designing stochastic memory cells [36] and logic gates [37] with bistable potentials, and the effectiveness of the potential-based design was demonstrated in robust operation under noisy

Table I. Dynamical systems design for nonlinear circuits and devices.

\begin{tabular}{|l|l|l|}
\hline Design Approaches & Target Devices and Circuits & Refs. \\
\hline \hline Phase Plane and Nullcline-based Design & & \\
& $\begin{array}{l}\text { Reaction Diffusion Chips } \\
\text { Silicon Neurons }\end{array}$ & $\begin{array}{l}{[34]} \\
{[28-33]}\end{array}$ \\
\hline Potential-based Design & Silicon Neurons & {$[35]$} \\
& Stochastic Memory Cells & {$[36]$} \\
& Stochastic Logic Gates & {$[37]$} \\
\hline Phase Response Curve-based Design & & \\
& Nonlinear Oscillator Circuits & {$[38,46]$} \\
& Spin Torque Nano Oscillators & {$[39]$} \\
\hline
\end{tabular}


environments.

In this work, we present the PRC-based design for SiNs as a dynamical systems design for SiNs to enhance synchronization in an ensemble of SiNs on the basis of the phase reduction theory [41]. By analyzing various circuit models of the previous SiNs [26-30], we explore key criteria to optimize the design of SiNs in terms of phase response properties. In particular, we focus on controlling transition among the Type I and Type II PRCs. In accordance with the criteria, as a case study, we demonstrate how to tune circuit parameters to obtain a desirable PRC of a resonate-and-fire neuron (RFN) circuit as an SiN [17], where only the peak location of the phase coupling function resulting from PRCs matters for phase locking.

The present paper is organized as follows. In Section 2, we clarify key criteria for the PRC-based SiN design through the analysis of the phase response properties of the various SiNs. In Section 3, we focus on the hybrid type SiNs for tractability and verify the validity of our design approach on the RFN circuit as a case study. We discuss a general design framework for the hybrid type SiNs in view of the phase reduction theory. In the last section, we present a conclusion.

\section{Dynamical systems design of SiN based on PRC}

To explore key design criteria of SiNs in PRC-based approach, we first capture essential features of phase response properties of SiNs through the comprehensive analysis of the PRCs of various SiNs. Then we derive a systematic design strategy for our approach from the results.

\subsection{Typical PRCs: Type I and Type II}

Before we consider specific examples of SiNs, we are reminded of two distinct PRCs of biological neurons [40]. In biological neurons, PRC quantifies the phase advance and delay in response to a perturbation during periodic firing. Two types of PRCs are identified in biological neurons. One is the Type I PRC that has almost all positive regions, which mean that the phase advances in response to a perturbation. Another one is the Type II PRC that has both positive and negative regions, which correspond to the phase advances and delays, respectively. Figure 1 shows the typical Type I and Type II PRCs. The classification of such PRCs is closely related to the bifurcation structure [40]. In the case of the saddle-node (on invariant circle) bifurcation, a neuron has the Type I PRC. In the case of both the supercritical/subcritical Andronov-Hopf bifurcation, a neuron has the Type II PRC. PRCs are useful in computational approaches for understanding entrainment and synchronization properties in coupled neural oscillators [42].

\subsection{Phase response properties of $\mathrm{SiNs}$}

We here consider essential features of the phase response properties of various kinds of SiNs. Since SiNs are designed by exploiting the nonlinear properties of constituting transistors, such as exponential voltage-current characteristics, which are quite different from biological ones, it is not trivial that PRCs of SiNs are qualitatively same as those of biological neurons. Therefore, it is needed to clarify specific features of the SiN's PRCs.
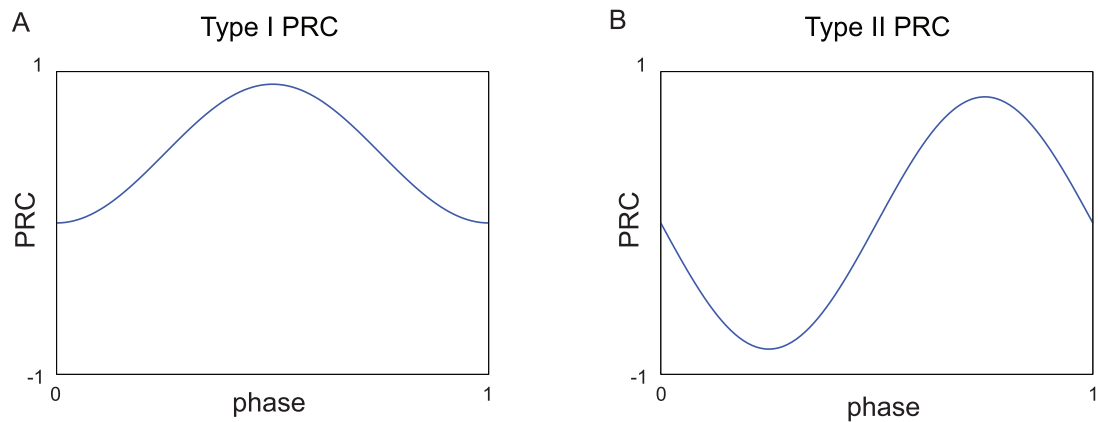

Fig. 1. Typical PRCs. (A) Type I and (B) Type II PRCs. 


\subsubsection{Integrate-and-fire (IFN) type SiNs}

Firstly, we show the phase response properties of the IFN type SiNs $[3,14-16,31-33]$. We begin by considering the relationship between the IFN type neuron models and their PRCs. The IFN type neuron models, such as the leaky IFN model and the quadratic IFN model, have the onedimensional subthrehold membrane dynamics with a firing threshold. Under constant current input beyond the critical current that causes the saddle-node bifurcation, the membrane voltage increases monotonically. Near the threshold voltage, the membrane voltage increases rapidly by the effect of the positive feedback. If the membrane voltage reaches the threshold voltage, the membrane voltage will instantaneously reset to a certain reset voltage by the effects of the negative feedback. Because of such dynamics, the IFN type neuron models have the Type I PRCs. For instance, the PRCs of the leaky IFN model and the quadratic IFN model can be analytically described as a function of the phase $\phi$ as $Z(\phi)=1-\exp (\beta \phi)(\beta=$ const.) and $Z(\phi)=1-\cos (\phi)$, respectively.

The shape of such PRCs are determined by the nonlinearity corresponding to the positive feedback. In fact, in the case of the leaky IFN model, the PRC is a monotonic exponential function with respect to the phase as described above. In contrast, in the case of the quadratic IFN model, the PRC is a nonmonotonic, positive convex upward function with respect to the phase. These facts indicate that the nonlinearity corresponding to the positive feedback is a key factor in the PRC-based design. To support this, we compare the PRCs of the cubic IFN model and the quadratic IFN model, because these models have been implemented on silicon [31-33]. Due to the difference in the nonlinearities, the maximum points of their PRCs are different, whereas the outlines of the PRCs are qualitatively similar [32].

\subsubsection{Full conductance-based type SiNs}

Next, we consider the phase response properties of the conductance-based type SiNs [24,25]. Since such conductance-based type SiNs have a wide variety of the conductance dynamics emulating biological ionic channels, the SiNs can have both Type I and Type II PRCs.

\subsubsection{Reduced conductance-based type SiNs}

We here show the phase response properties of a class of reduced conductance-based type SiNs, i.e., the Morris-Lecar (ML) type SiNs [26-30]. The dynamics of the ML neuron model can be derived from the conductance-based neuron models of the Hodgykin-Huxley (HH) type by model reduction. We consider the reduced dynamics with two state variables as follows:

$$
\begin{aligned}
\tau \frac{d v}{d t} & =f(v, w, i) \\
\frac{d w}{d t} & =g(v, w)
\end{aligned}
$$

where $v$ and $w$ represent the state variables corresponding to the fast membrane and slow recovery dynamics, respectively. The time constant $\tau$ can tune the time scale of the system dynamics. The input current $i$ can be regarded as a bifurcation parameter.

In the previous works, several SiNs modeled after the dynamics of the ML type neuron models have been proposed [26-30]. To clarify specific features of phase response properties of such SiNs, we numerically computed the infinitesimal PRCs (iPRCs), which are equivalent to the phase sensitivity functions, by using the adjoint method [40].

In the pioneer work, the first ML type SiN was presented to model biological synchrony transitions [26]. The dynamics of the SiN are represented as follows:

$$
\begin{aligned}
C_{V} \frac{d V}{d t} & =I \alpha_{P}+I_{B H} \frac{e^{\kappa\left(V-V_{H}\right) / U_{T}}}{1+e^{\kappa\left(V-V_{H}\right) / U_{T}}} \alpha_{P}-I_{B L} \frac{e^{\kappa\left(W-W_{L}\right) / U_{T}}}{1+e^{\kappa\left(W-W_{L}\right) / U_{T}}} \alpha_{N} \\
C_{W} \frac{d W}{d t} & =I_{\tau} \tanh \left(\kappa(V-W) / U_{T}\right) \beta_{P} \beta_{N}
\end{aligned}
$$

where the functions $\alpha_{P}=1-e^{\kappa\left(V-V_{H i}\right) / U_{T}}, \alpha_{N}=1-e^{\kappa\left(V_{L o}-V\right) / U_{T}}, \beta_{P}=1-e^{\kappa\left(W-V_{D D}\right) / U_{T}}$, $\beta_{N}=1-e^{\kappa(-W) / U_{T}} . V$ and $W$ are the node voltages that represents the state variables corresponding 

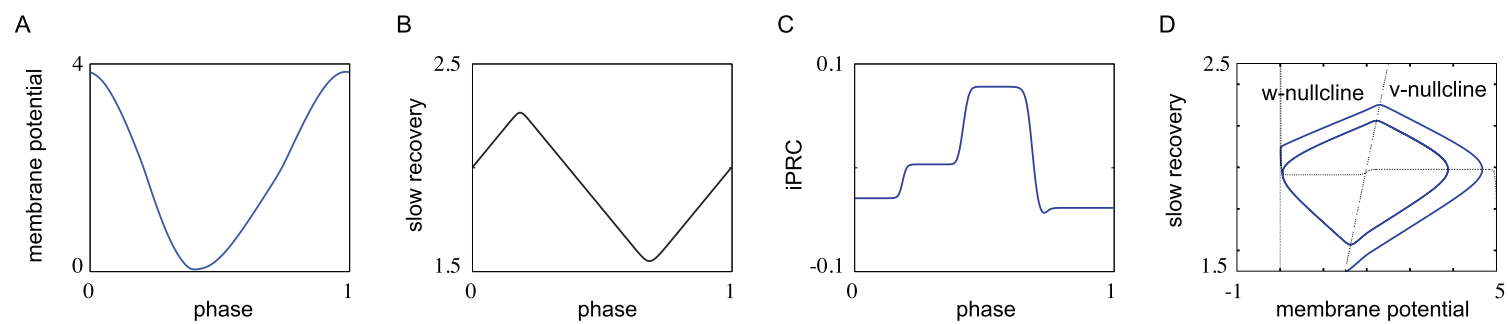

Fig. 2. Dynamical properties of the first ML type SiN. (A) Fast membrane and (B) slow recovery dynamics. (C) iPRC. (D) Phase plane portrait.
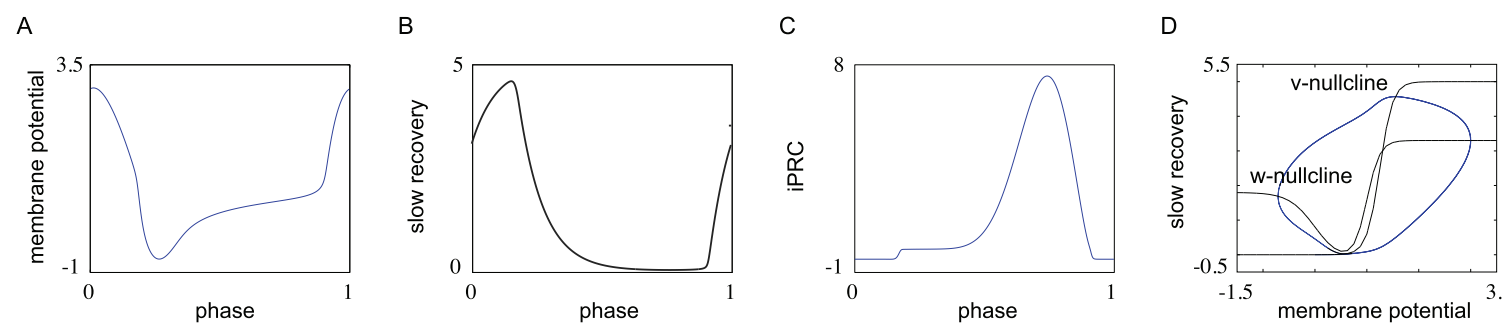

Fig. 3. Dynamical properties of the second ML type SiN. (A) Fast membrane and (B) slow recovery dynamics. (C) iPRC. (D) Phase plane portrait.
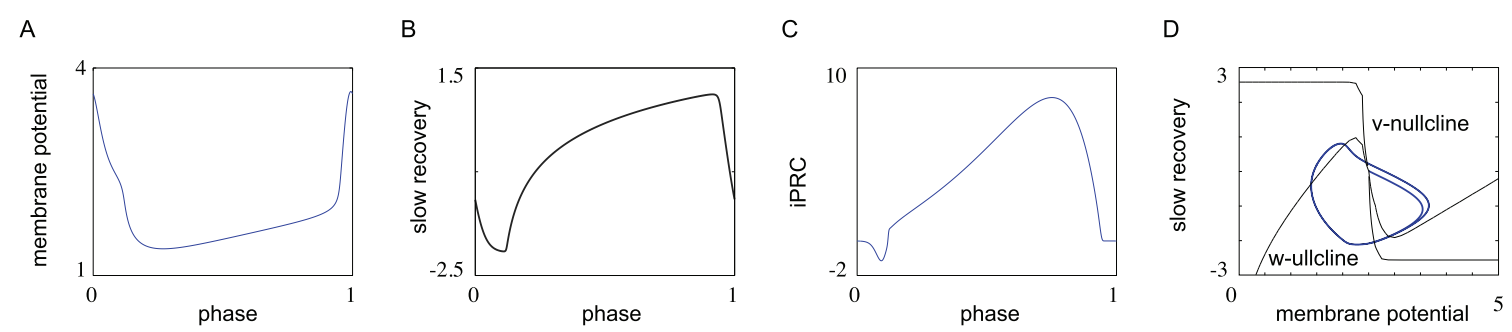

Fig. 4. Dynamical properties of the third ML type SiN. (A) Fast membrane and (B) slow recovery dynamics. (C) iPRC. (D) Phase plane portrait.

to the membrane dynamics and the slow recovery dynamics, respectively. The parameters $V_{H}, V_{L}$, $V_{H i}, V_{L o}$ are the bias voltages, and $\kappa$ the subthreshold slope factor and $U_{T}$ the thermal voltage, which are determined by the fabrication process. Depending on the power-supply voltages $V_{D D}$ and the bias voltages, the circuit can operate in the high-voltage mode and the low-voltage mode. The input current $I$ works as a bifurcation parameter, the bias currents $I_{B H}$ and $I_{B L}$ determine the dynamic range, and $I_{\tau}$ can tune the time constant. The capacitances $C_{V}$ and $C_{W}$ determine the time scale of the dynamics.

Figure 2 shows the dynamics and the PRCs of the SiN. Since the dynamic range of the voltages are restricted by the bias voltages, the top and bottom of the PRC for the $V$-dynamics are flat. The phase plane portrait rotates in a counterclockwise direction.

The second ML type SiN, an SiN circuitry with the Class 1 and Class 2 properties has been derived, as a case of the nullcline and phase plane-based design, by qualitative neuronal modeling using a model reduction technique [28]. Besides the original circuit was designed as a three dimensional fast-slow system, we hereafter focus on the dynamics of the fast subsystem of the SiN, which are represented as follows:

$$
\begin{aligned}
C_{V} \frac{d V}{d t} & =-g(V)+f_{V}(V)-W+I \\
\frac{d W}{d t} & =\frac{f_{W}(V)-W}{\tau_{W}}
\end{aligned}
$$

with

$$
f_{x}(v)=M_{x} \frac{1}{1+\exp \left(-\frac{\kappa}{U_{T}}\left(v-\delta_{x}\right)\right)}
$$




$$
g(v)=S \frac{1-\exp \left(-\frac{\kappa}{U_{T}}(v-\theta) / 2\right)}{1+\exp \left(-\frac{\kappa}{U_{T}}(v-\theta) / 2\right)}
$$

where $V$ and $W$ are the node voltage and the current that represents the state variables corresponding to the membrane dynamics and the slow recovery dynamics, respectively. The current $I$ includes both input current and bias constant current. The nonlinear functions $f_{x}(v)(x=V, W)$ and $g(v)$ can be implemented by the differential pair operating in the subtheshold region. The coefficients $M_{x}$ and $S$ can be determined by the bias currents of such differential pairs and the parameters $\delta_{x}$ and $\theta$ represent the bias voltages of the differential pairs. On the phase plane, the portrait rotates in a counterclockwise direction. Figure 3 shows that the PRC becomes the Type II having a negative region, which increases with the distance from the saddle-node bifurcation point.

The third ML type SiN, an SiN circuitry with the Class 1 excitability has been designed [30] based on the biophysically-inspired SiN with the Class 2 excitability [27]. Since the latter one has the four-dimensional dynamics, we focus on the former one reduced to the two-dimensional dynamics. By changing circuit configuration for tuning phase plane structure, the saddle-node bifurcation on invariant circle and the saddle-node homoclinic bifurcation can occur in the SiN.

The dynamics of the saddle-node SiN are represented as follows:

$$
\begin{aligned}
\frac{d V}{d t} & =e^{-M_{s s}(V)}\left(1-e^{V-8}\right)-I_{k 0} e^{-W}\left(e^{V}-1\right)-I_{l 0}\left(1-e^{E l-V}\right)+I \\
\frac{d W}{d t} & =r\left(e^{-W}-e^{-N_{s s}(V)}\right)
\end{aligned}
$$

where $V$ and $W$ represent the normalized voltages and $I$ the normalized current obtained by the transformation of variables. Other parameters are derived from the normalization of the circuit parameters. The nonlinear functions are defined as follows: $M_{s s}(V)=-\alpha_{1} \tanh \left(\left(V-o_{1}\right) / s_{1}\right)$ and $N_{s s}(V)=-\alpha_{2} \tanh \left(\left(V-o_{2}\right) / s_{2}\right)$.

Figure 4 shows the circuit dynamics and the PRC of the SiN. The dynamics of the SiN are different from those of the other SiNs mentioned above with respect to the slow recovery variable. Since the polarity of the recovery dynamics is opposite, the phase plane portraits rotate in a clockwise direction. In spite of near the saddle-node bifurcation point, there exist a narrow negative region corresponding to the Type II PRC. The area of such a negative region depends on the gradient and duration of the negative feedback, indicating the significance of controlling the negative feedback.

From the viewpoint of the bifurcation theory, the existence of the negative region can be well explained. Since the saddle-node neuron has the Hopf bifurcation point as well as the saddle-node bifurcation point [30], the phase response properties of the SiN are affected by both the bifurcation. In fact, the area of the negative region increases with distance from the saddle-node bifurcation point by current injection. The results are consistent with the relationship between the type of PRCs and the bifurcation transitions found in biological neuron models [47]. This indicates the significance of controlling global bifurcation transitions to obtain a desirable PRC in designing the reduced conductance-based SiNs. However, it is quite difficult to control transition between Type I and Type II PRCs by switching a circuit parameter without changing the input current as a bias parameter.

\subsubsection{Hybrid type SiNs}

We here consider the phase response properties of the hybrid type SiNs that have both continuous subthreshold membrane dynamics and discontinuous firing reset mechanism. For the purpose, we compare the phase plane dynamics and phase response properties of the Izhikevich neuron model and the RFN model [40].

Figure 5 shows the phase plane portraits of the Izhikevich model. Depending on the bifurcation near the equilibrium point, the Izhikevich model can exhibit both the Type I and Type II PRCs, as shown in Fig. 6. In the case of near the saddle-node bifurcation (Fig. 5A), the Izhikevich model becomes an integrator having the Type I PRC (Fig. 5A). In contrast, in the case of near the Hopf bifurcation (Fig. 6B), the Izhikevich model becomes a resonator having the Type II PRC (Fig. 6B). 
A

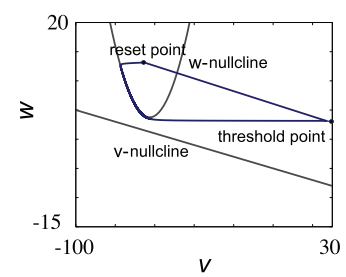

B

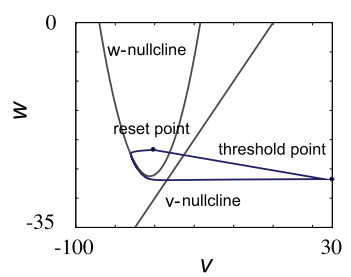

A

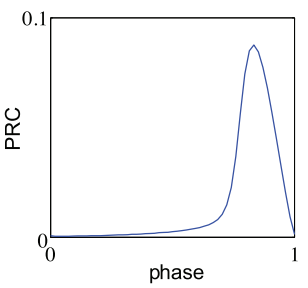

B

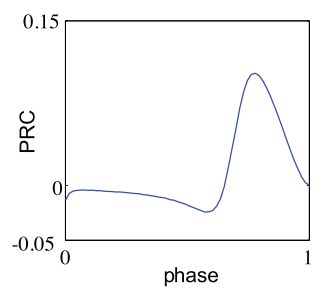

Fig. 5. Phase plane portraits of the Izhikevich neuron model in the case of $(\mathrm{A})$ integrator and $(\mathrm{B})$ resonator.

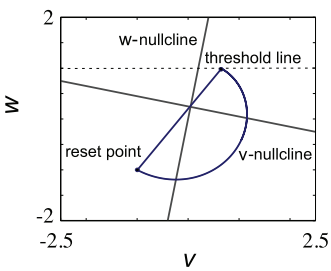

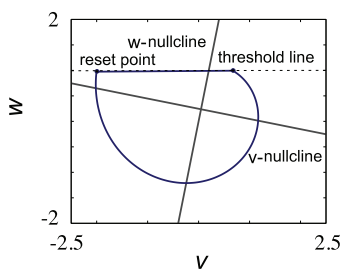

Fig. 7. Phase plane portraits of the resonate-and-fire (RFN) neuron model.

Fig. 6. PRCs of the Izhikevich neuron model in the case of $(\mathrm{A})$ integrator and $(\mathrm{B})$ resonator.
A

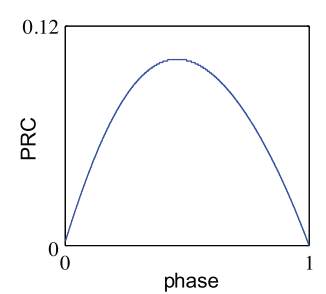

B

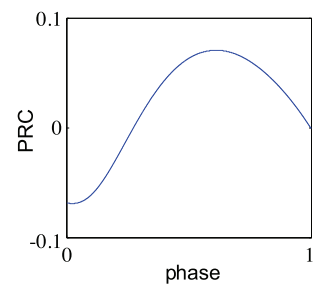

Fig. 8. PRCs of the RFN model. (A) Type I and Type II PRCs.

By changing the ratio of the time constants of the membrane dynamics $(v)$ and the recovery dynamics $(w)$ or the location of the reset point of the phase plane, the shape of the PRC can be modified.

Figure 7 shows the phase plane portraits of the RFN model. Depending on the parameters, the model has a stable focus and exhibits damping subthreshold oscillation. After the grazing bifurcation beyond a threshold, the RFN model fires a spike and the state is reset to a certain reset point.

The PRCs of the RFN model can be analytically represented as in the following form [45]:

$$
Z(\phi)=\gamma \exp (T \phi) \sin \left(2 \pi \frac{T}{T_{o}}(1-\phi)\right)
$$

where $\phi$ represents the phase variable, $T$ the repetitive firing period, and $T_{o}$ the maximum firing period inversely proportional to the natural angular frequency of the RFN model. Near the grazing bifurcation point, $T$ becomes close to $T_{o}\left(T<T_{o}\right)$. The coefficient $\gamma$ can be exactly calculated in the phase reduction analysis of the RFN model [45]. Since the repetitive firing period depends on the relative position of the reset point to the threshold on the phase plane, the shape of the PRC can be changed by setting the location of the threshold line and the reset point (Fig. 8). In fact, the region of the negative phase shift corresponding to the Type II PRC becomes large with the increasing the angle between the threshold line and the reset point from the stable focus as shown in Fig. 8.

This result suggests that one can easily control the transition between the Type I and Type II PRCs by changing the location of the reset voltage relative to the threshold. In a similar fashion, the adaptation mechanism of the exponential integrate-and-fire neuron model can change its PRC [43]. These results indicate the significance of tuning the location of the reset point as well as the threshold in the phase plane.

\subsection{Systematic design strategy derived from meta-analyses of SiN PRCs}

We derive a systematic strategy for the PRC-based design of SiNs from the extensive meta-analysis in this section. In our design strategy, the following STEP1 and STEP2 are preliminary procedures for STEP3. Since the main purpose of our design presented here is to control the transitions among the Type I and Type II PRCs, STEP3 is the main part of our design strategy.

As assumptions, an SiN to design has been determined and its circuit topology (configuration) is given. This means that our ultimate goal is fine-tuning all parameters to realize desirable PRCs. It should be noted that design variables (parameters) of SiNs can be divided into two: circuit parameters (for instance, capacitance, internal resistance of transistors, and the thermal voltages etc.) and bias parameters (for instance, bias voltages and bias currents). It is difficult to change the circuit 
parameters after design and fabrication, whereas the bias parameters can be controlled dynamically even during circuit operation. This implies that the bias parameters can only be used as bifurcation parameters. Therefore, it is not so easy to control global bifurcation in a given SiN after design and fabrication. In short, the highlight of our design is that the SiN should be designed to be tractable so that even a few bias parameter can control the global bifurcation, leading to the transitions among Type I and Type II PRCs.

STEP1: First, we tune the nullcline curves and set the location of the equilibrium point on the phase plane to determine the bifurcation type near the equilibrium point, for instance, a saddle-node on invariant circle bifurcation, a superctitical Andronov-Hopf bifurcation, and other bifurcation, in accordance with the phase plane and nullcline-based design.

In the approach, qualitative visualisation of nullcline curves provide a cue to finding a parameter set to realize a desirable local vector field in a systematic way. For instance, in the cases of Figs. 2 to $4, v$-nullclines are simple sigmoidal curves and $w$-nullcines are more complicated non-monotonous curves. It is relatively difficult to modify $w$-nullcines as compared with $v$-nullclines. By changing the parameters, one can modify such nullcline curves and the location of equilibrium points as intersection points of these curves. Topological features of nullcline curves, such as hyperbolicity, the number of intersection points, and curvature near the equilibrium points, provide a key to designing a local vector field.

Plotting a phase plane portrait is useful for designing a global vector field. Furthermore, we are used to computing and plotting a global vector field numerically using XPPAUT.

In addition to these means in the phase plane and nullcline-based design approach, in our approach, we focus on PRC as mathematical structure of an SiN, providing a key to capturing a global vector field of the SiN. Consequently, the bifurcation type near an equilibrium point of an $\mathrm{SiN}$ can be determined.

STEP2: Second, we modify all the parameters to achieve a balance between (i) controlling both positive and negative feedback gains and (ii) tuning the ratio between the duration of action potential and the repetitive firing period. In practice, tuning the time constant of the circuit dynamics is essential for (i) and (ii). It should be noted that as an assumption it is hard to rescale the time axis in SiNs after fabrication. Thus, it is necessary to balance among circuit and bias parameters in circuit design before fabrication. For instance, the time constants of the circuit dynamics are determined by the balance among capacitances and current flow. If once the capacitances were fixed, and then we can only change the current flow.

STEP3: Next, we care about (iii) controlling the transition between type Type I and Type II PRCs. Here we consider to control the transition between type Type I and Type II PRCs by dynamically changing the bias parameters, such as bias voltages and bias currents. It should be noted that no circuit parameter can be used as a control parameter after design and fabrication. Thus, it is not so simple to obtain the intersection between two parameter sets resulting the Type I and Type II PRCs.

We hereafter consider how to control the transition between Type I and Type II PRCs in specific SINs. In the case of the ML Type SiNs as the reduce conductance-based SINs, one can use the bias current that acts as an input current to the $\mathrm{SiN}$ as a control parameter as described in 2.2.3. In contrast, in the case of the Hybrid Type SINs, one can use both the reset voltage and threshold voltage as a control parameter as described in 2.2.4. These bias parameters are independent of the input current. In this sense, the Hybrid Type SINs are more tractable than the ML Type SiNs. Therefore, we will focus on the design of the hybrid type SiNs in the next section.

\section{Practical design of a hybrid type SiN}

Let us demonstrate practical design of a hybrid type SiN, the RFN circuit, based on PRCs. We explain how to tune circuit parameters of the RFN circuit in accordance with our design criteria. 
The RFN circuit can satisfy (i), (ii) and (iii) mentioned in the previous section due to the firing reset mechanism corresponding to precipitous negative feedback and the subthreshold membrane dynamics leading to the grazing bifurcation.

\subsection{The resonate-and-fire neuron circuit}

Figure 9 shows the schematic diagram of the RFN circuit that consists of the membrane circuit, the threshold-and-fire circuit, and the excitatory and inhibitory synaptic circuits. The dynamics of the RFN circuit are represented as:

$$
\begin{aligned}
C_{V} \frac{d V}{d t} & =-g V+\dot{I}_{V}-I_{o} \exp \left(\frac{\kappa^{2}}{\kappa+1} \frac{W}{U_{T}}\right)+I \\
C_{W} \frac{d W}{d t} & =I_{o} \exp \left(\frac{\kappa^{2}}{\kappa+1} \frac{V}{U_{T}}\right)-\dot{I}_{W}
\end{aligned}
$$

with

$$
\begin{aligned}
& \dot{I}_{V}=\alpha I_{V}\left(1+\frac{V_{D D}-V}{V_{E, P}}\right) \\
& \dot{I}_{W}=\beta I_{W}\left(1+\frac{W}{V_{E, N}}\right)
\end{aligned}
$$

where $V$ and $W$ are the node voltages that represents the state variables corresponding to the membrane dynamics and the slow recovery dynamics, respectively. The currents $I_{V}$ and $I_{W}$ are transferred to $\dot{I}_{V}$ and $\dot{I}_{W}$ through the current-mirrors that consist of the transistors with short channels. The parameters $V_{E, N}$ and $V_{E, P}$ correspond to the Early voltages of the NMOS and PMOS FETs, respectively. The current $I_{0}$ represents the pre-exponential current of the transistor operating in the weak inversion region.

If the voltage $W$ reaches at the threshold voltage $V_{t h}$ by current injection, the pules signal $V_{\text {pulse }}$ is generated and converted into the reset signal $V_{\text {res }}$ through the delay-and-inverter circuit (Fig. 10). The reset signal is fed into the membrane circuit for resetting the state, $(V, W)$, to the reset voltages, $\left(V_{\text {rst }}, W_{\text {rst }}\right)$. Figure 11 shows the phase plane portraits of the RFN circuit, in which we set the reset voltages $\left(V_{\text {rst }}, W_{\text {rst }}\right)$ to $(0.74 \mathrm{~V}, 0.75 \mathrm{~V})$ for Fig. $11 \mathrm{~A}$ and $(0.80 \mathrm{~V}, 0.74 \mathrm{~V})$ for Fig. $11 \mathrm{~B}$, respectively.

By substituting the circuit parameters into Eq. (11), we can obtain the PRC of the RFN circuit.

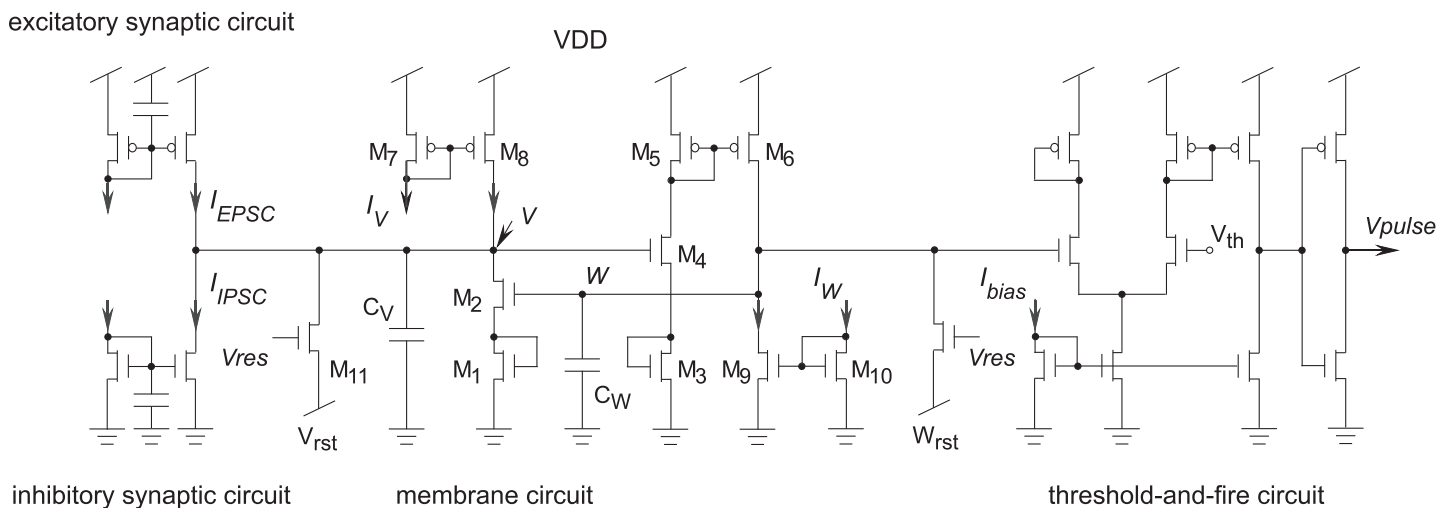

Fig. 9. Schematic of the RFN circuit that consists of a membrane circuit, a threshold-and-fire circuit, and excitatory and inhibitory synaptic circuit.

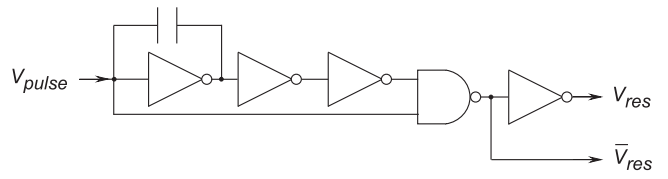

Fig. 10. Schematic of the delay-and-inverter circuit. 


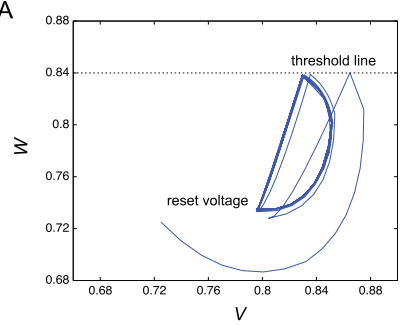

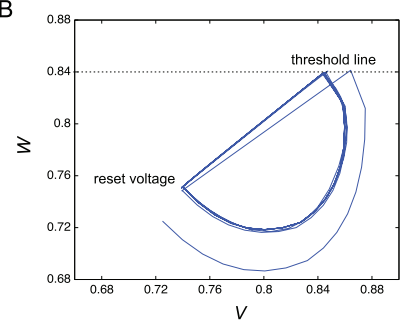

Fig. 11. Phase plane portraits of the RFN circuit, in which the reset voltages $\left(V_{\mathrm{rst}}, W_{\mathrm{rst}}\right)$ were set to $(\mathrm{A})(0.74 \mathrm{~V}, 0.75 \mathrm{~V})$ and $(\mathrm{B})(0.80 \mathrm{~V}, 0.74 \mathrm{~V})$.

The natural angular frequency of the RFN circuit can be computed by the Jacobian matrix around at the equilibrium point of the circuit as follows [17]:

$$
J=\left[\begin{array}{cl}
-\frac{\alpha I_{V}}{V_{E, P}} & -\frac{\kappa^{2}}{\kappa+1} \frac{I_{W_{o}}}{U_{T}} \\
\frac{\kappa^{2}}{\kappa+1} \frac{I_{V_{o}}}{U_{T}} & -\frac{\beta I_{W}}{V_{E, N}}
\end{array}\right]
$$

where $I_{U_{o}}$ and $I_{V_{o}}$ represent the equilibrium currents at the equilibrium point $\left(V_{o}, W_{o}\right)$. By assuming the equilibrium currents $I_{U_{o}}$ and $I_{V_{o}}$ are same, the natural angular frequency of the circuit can be represented as $\omega=\frac{\kappa^{2}}{\kappa+1} \frac{I_{V_{o}}}{U_{T}}$.

\subsection{Synchronization phenomena in the RFN circuit network}

We verified synchronization properties of fully pulse-coupled networks of the RFN circuits with a circuit simulator, SPICE. Through the following simulations, we set a common parameter set as follows: $I_{\text {bias }}=250 \mathrm{nA}, V_{\mathrm{th}}=0.84 \mathrm{~V}, V_{\mathrm{rst}}=0.75 \mathrm{~V}$, and $W_{\mathrm{rst}}=0.80 \mathrm{~V}$. The number of the circuits was set as $N=5$. The device parameters were assumed to use the TSMC $0.25-\mu \mathrm{m}$ technology.

\subsubsection{Delay-induced synchronization in excitatory-connected network}

First, we investigated synchronization properties of an excitatory-connected network of the RFN circuits. We set the capacitance $C_{V}=C_{W}=0.25 \mathrm{pF}$ and the bias current $I_{V}=I_{W}=15.0 \mathrm{nA}$ to obtain the Type II PRC.

In the case of small transmission delays, the network exhibits an out-of-phase synchronization with uniform phase distribution, so-called a merry-go-round state, as shown in Fig. 12A. In contrast, in the case of relatively large transmission delays beyond a certain value, the circuits synchronize in in-phase at a steady state, i.e., an in-phase locked synchronization state, as shown in Fig. 12B. The critical value for the in-phase locked state can be calculated by the network linear stability analysis presented in Appendix. The theoretical analysis indicates that the location of the extrema of the phase coupling function is crucial for inducing the transition between the in-phase locked and out-of-phase synchronization states. That is, when the transmission delay is longer than the peak location of the resulting phase coupling function, the in-phase locked state is stable. To confirm this, we measured the PRC and the phase coupling function resulting from the interaction between the PRC and the synaptic current, as shown in Fig. 13. Since the synaptic current cannot be represented in the explicit mathematical form, we directly measured the phase coupling function by using a perturbation via the synaptic circuit. The result is consistent with the theoretical implication.

In practice, synaptic conductance and transmission delays are unable to disregard in silicon spiking neural networks in large-scale. In view of this situation, delay-induced synchronization demonstrated here is useful for compensating such delays for global synchronization at network level.

\subsubsection{The out-of-phase synchronization states in both excitatory- and inhibitory- connected networks}

Next, we demonstrate the existence of the out-of-phase synchronization states in both excitatory- and inhibitory-connected networks of the RFN circuits, as shown in Figs. 14A and B, respectively. Here 
A
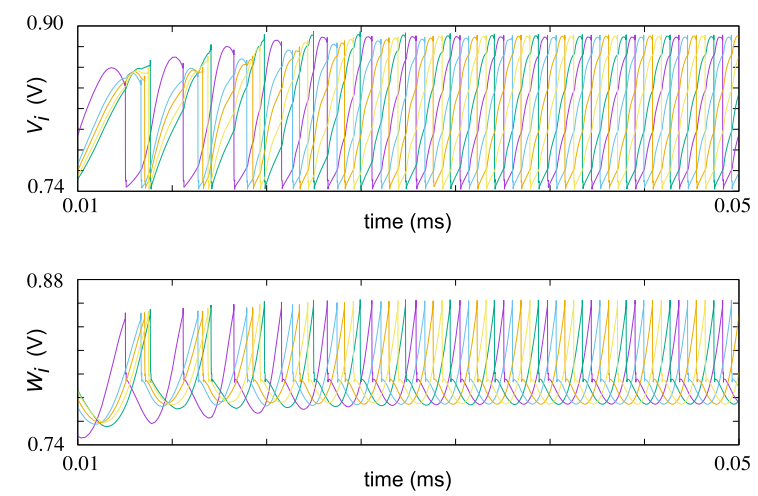

B
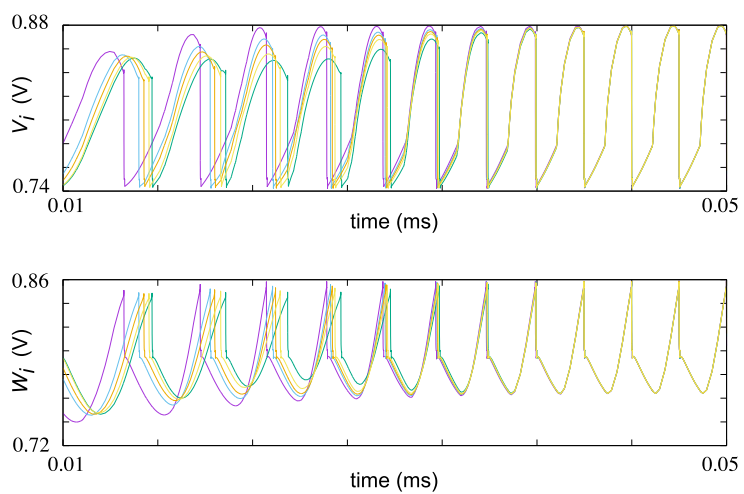

Fig. 12. Delay-induced synchronization in excitatory-connected network. (A) An out-of-phase synchronization state without delay. (B) An in-phase locked synchronization state with large delay.

A

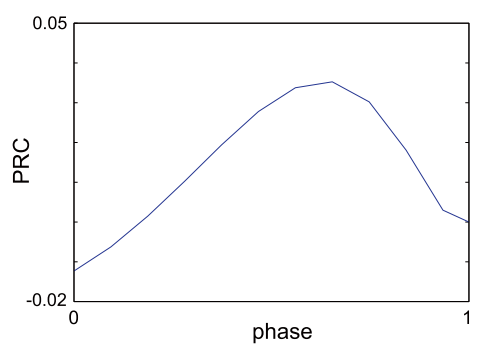

B

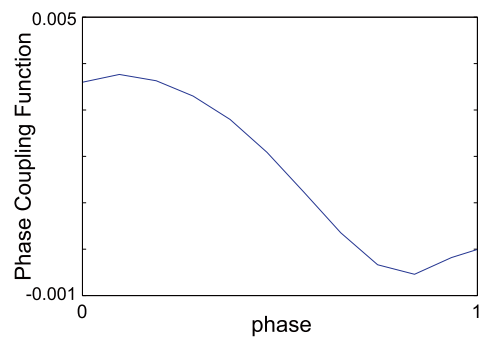

Fig. 13. Phase response properties of the RFN circuit. (A) PRC and (B) phase coupling function.

we set the capacitance $C_{V}=C_{W}=1.0 \mathrm{pF}$ and the bias current $I_{V}=I_{W}=22.5 \mathrm{nA}$ for the excitatoryconnected network and $C_{V}=C_{W}=1.5 \mathrm{pF}$ and $I_{V}=I_{W}=19.0 \mathrm{nA}$ for the inhibitory-connected network.

From the viewpoint of the phase reduction theory, we consider the occurring condition for such out-of-phase synchronization states. The complicated linear stability analysis of the out-of-phase synchronization state, especially in the limit of large number of neurons, has been studied in the literatures [44]. In the case of the limited number of neurons, for the out-of-phase synchronization states, the linear stability analysis of a map presented in Appendix gives only the necessary condition that no synchronization state is stable. The measured phase coupling functions (Figs. 15A and B) satisfy this necessary condition. In practice, we changed the coupling strength of the synaptic circuits to find a desirable out-of-phase synchronization state under the necessary condition. Depending on the coupling strength, a different number of the RFN circuits can exhibit the out-of-phase synchronization states, such as shown in Fig. 16, in which the synaptic strength were set as (A) $480 \mathrm{nA}$ and (B) $550 \mathrm{nA}$, respectively. Further phase reduction analysis may enhance the network design for the out-of-phase synchronization states.

\section{Conclusion and discussion}

We have extensively considered the PRC-based design for SiNs. By analyzing various circuit models of the SiNs, we clarified the key criteria for optimization of the design of SiNs in terms of phase response properties. As a case study, we demonstrated how to tune the circuit parameters of the RFN circuit to obtain a desirable PRC in accordance with our design criteria. More specifically, we showed how to tune the circuit parameters of the RFN circuit as a hybrid type SiN to obtain both Type I and Type II PRCs. Furthermore, we demonstrated that setting the location of maxima of the PRC of the RFN circuit is crucial for controlling both in-phase locked and out-of-phase synchronization states in a network of the RFN circuits with transmission delay. It is useful to control transition between the Type I and Type II PRC for setting the location of extrema of the PRC. The results indicate the 

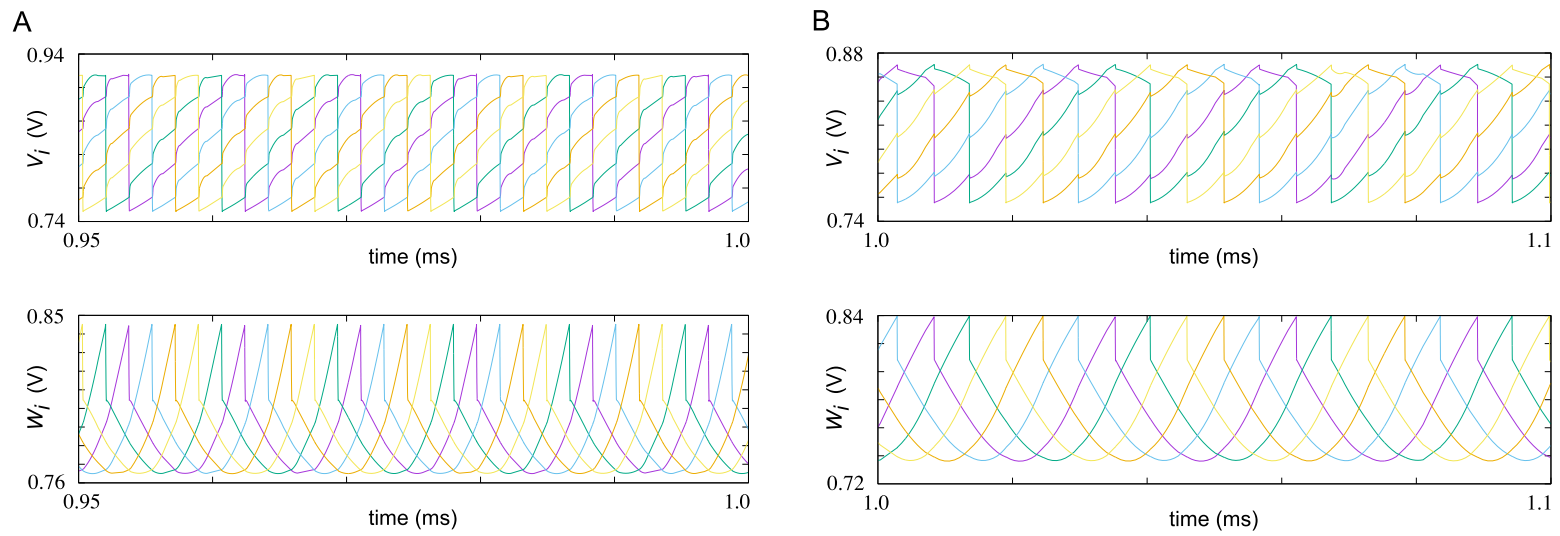

Fig. 14. The out-of-phase synchronization states in the cases of (A) excitatory- and (B) inhibitory-connected networks.

A

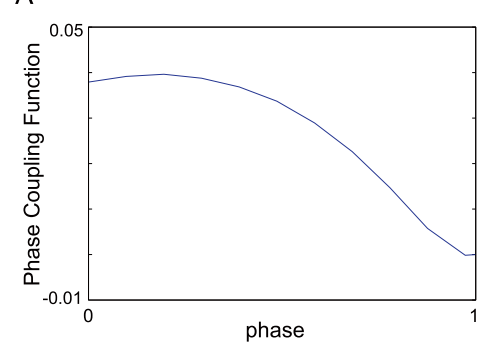

B

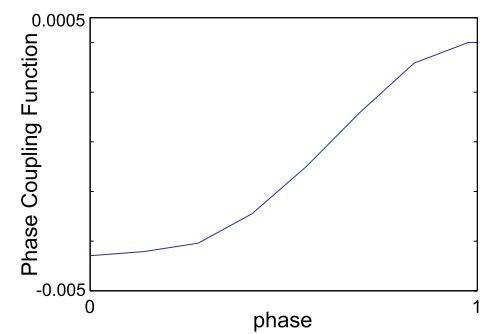

Fig. 15. Measured phase coupling functions corresponding to out-of-phase synchronization states in the cases of (A) excitatory- and (B) inhibitoryconnected networks.

A
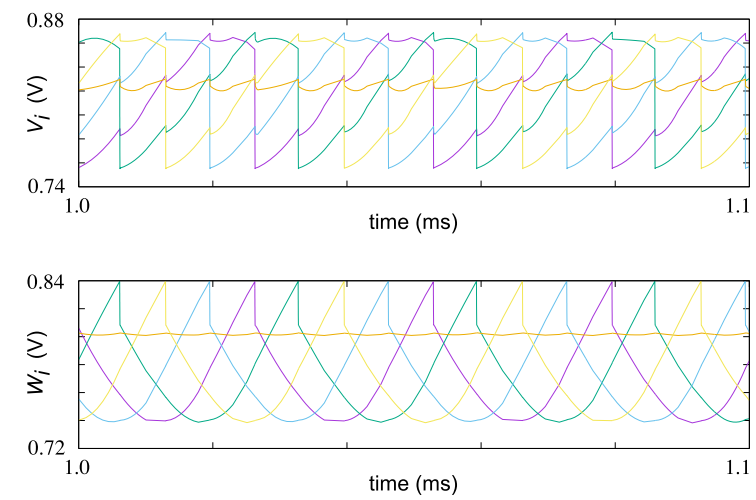

B
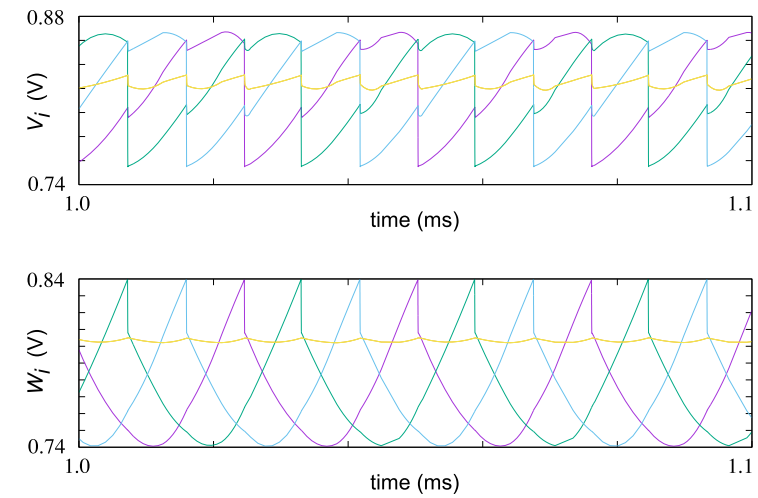

Fig. 16. The out-of-phase synchronization states in inhibitory-connected networks, in which the synaptic strength were set as (A) $480 \mathrm{nA}$ and (B) $550 \mathrm{nA}$, respectively.

significance of our approach in practical SiN design.

In neuromorphic systems using spike-based computation, controlling synchronization states plays significant roles in various information processing. For instance, the out-of-phase synchronization states demonstrated here suggest that neuromorphic noise shaping can be realized in both excitatoryand inhibitory-connected networks of SiNs by tuning the PRCs of the individual SiN. The PRC-based design approach presented here can enhance optimization of such network design.

Our design approach plays a complementary role to the other dynamical systems design approaches and provides us with a unified perspective in SiN design. Since our approach can be applicable to design a wide variety of SiNs, we are going to extend our approach to enhance functional synchronization in the large-scale network of various SiNs towards future cognitive neuromorphic computing exploiting spike-based computation. 


\section{Appendix}

\section{A. Linear stability analysis of global synchronous states}

Let us here demonstrate the linear stability analysis of global synchronous states, i.e., in-phase locked synchronization states. In the following analysis, we assume that synaptic current couplings among SiNs are instantaneous interactions and discontinuous in time.

\section{A.1 Derivation of linearized equation}

We consider all-to-all delayed coupled oscillators:

$$
\frac{d \phi_{i}}{d t}=\omega_{0}+\frac{K}{N} \Gamma\left(\phi_{i}\right) \sum_{k} \delta\left(t-t_{k}-d\right)
$$

where $\phi_{i}$ represents the phase variable of $i$-th oscillator ( $N$ the number of the oscillators), $\omega_{0}$ the natural angular frequency, $K$ coupling strength, $t_{k}$ the last firing time of the $k$-th oscillator, and $d$ the transmission delay. The phase coupling function $\Gamma[41]$ is defined as:

$$
\Gamma(\phi)=\frac{1}{2 \pi} \int_{0}^{2 \pi} Z(\phi+\psi) p(\psi) d \psi
$$

where $Z$ represents the phase sensitivity function (infinitesimal PRC). Here, the function $p$ is a synaptic function corresponding to a perturbation. In a special case that the synaptic function can be modeled as the delta function, $\Gamma(\phi)$ is just equivalent to the phase sensitivity function, $Z(\phi)$.

When the system is almost completely synchronized, the state can be written as:

$$
\phi_{i}(t)=\omega t+\Delta_{i}(t)
$$

where $\omega$ is the frequency of the coupled system and $\Delta$ is small. Then, after the time-averaging for a period.

$$
\begin{aligned}
\Delta_{i}((n+1) T) & =\Delta_{i}(n T)+\frac{K}{N} \sum_{j} \Gamma\left(d+\Delta_{i}(n T)-\Delta_{j}(n T)\right)-\Gamma(d) \\
& \approx \Delta_{i}(n T)+\frac{K}{N} \sum_{j} \Gamma(d)^{\prime}\left(\Delta_{i}(n T)-\Delta_{j}(n T)\right) .
\end{aligned}
$$

This can be rewritten in the matrix form,

$$
\Delta((n+1) T)=A \Delta(n T)
$$

where

$$
A=I+K \Gamma(d)^{\prime}\left(\begin{array}{cccc}
N & -1 & \ldots & -1 \\
-1 & N & \ldots & -1 \\
-1 & -1 & \ddots & -1 \\
-1 & -1 & \ldots & N
\end{array}\right) / N
$$

\section{A.2 Linear stability based on eigenvalues}

The eigenvalues of $A$ are $\left(1,1+K \Gamma(d)^{\prime}, 1+K \Gamma(d)^{\prime}, \ldots, 1+K \Gamma(d)^{\prime}\right)$. So, if $K \Gamma(d)^{\prime}$ is small and negative $\left(\left|1+K \Gamma(d)^{\prime}\right|<1\right)$, the in-phase locked synchronization state is stable. This suggests that the in-phase locked synchronization state can destabilize when the PRC is steep or the coupling is strong enough.

\section{References}

[1] C. Mead, Analog VLSI and neural systems, Addison-Wesley, 1989.

[2] M. Mahowald and R. Douglas, "A silicon neuron," Nature, vol. 354, pp. 515-518, 1991.

[3] S.-C. Liu, J. Kramer, G. Indiveri, T. Delbruck, and R. Douglas, Analog VLSI: Circuits and principles, The MIT press, 2002. 
[4] T. Lande, Neuromorphic systems engineering: neural networks in silicon, vol. 447, Kluwer Academic Pub., 1998.

[5] S.-C. Liu and T. Delbruck, "Neuromorphic sensory systems," Current Opinion in Neurobiology, vol. 20, no. 3, pp. 288-295, 2010.

[6] F. Tenore, R. Etienne-Cummings, and M.A. Lewis, "Entrainment of silicon central pattern generators for legged locomotory control," in Proc. of Neural Information Processing Systems, vol. 16, S. Thrun, L. Saul, and B. Scholkopf (Eds.), MIT Press, Cambridge, MA, 2004.

[7] P. Arena, A. Basile, L. Fortuna, M. Frasca, and L. Patané, "Implementation of Turing patterns for bio-inspired motion control," in Proc. IEEE ISCAS, vol. 3, pp. 842-845, 2003.

[8] K. Nakada, T. Asai, and Y. Amemiya, "Analog CMOS implementation of a CNN-based locomotion controller with floating-gate devices," IEEE Trans. Circuits and Systems I, vol. 52, no. 6, pp. 1095-1103, 2005.

[9] F. Tenore and R. Etienne-Cummings, "Biomorphic circuits and systems: Control of robotic and prosthetic limbs," in Proc. IEEE Biomedical Circuits and Systems Conference, pp. 241$244,2008$.

[10] J. Barron-Zambrano, C. Torres-Huitzil, and B. Girau, "Hardware implementation of a CPGbased locomotion control for quadruped robots," in Proc. ICANN, pp. 276-285, 2010.

[11] T. Delbruck and P. Lichtsteiner, "Fast sensory motor control based on event-based hybrid neuromorphic-procedural system," in Proc. IEEE ISCAS, pp. 845-848, 2007.

[12] F. Gomez-Rodriguez, A. Linares-Barranco, L. Miro-Amarante, S.-C. Liu, A. van Schaik, R. Etienne-Cummings, and M.A. Lewis, "AER auditory filtering and CPG for robot control," in Proc. IEEE ISCAS, pp. 1201-1204, 2007.

[13] P.A. Merolla et al., "A million spiking-neuron integrated circuit with a scalable communication network and interface," Science, vol. 345, no. 6197, pp. 668-673, 2014.

[14] G. Indiveri, B. Linares-Barranco, T.J. Hamilton, A. van Schaik, R. Etienne-Cummings, T. Delbruck, S.C. Liu, P. Dudek, P. Häfliger, S. Renaud, and others, "Neuromorphic silicon neuron circuits," Frontiers in Neuroscience, vol. 5, 2011.

[15] E. Culurciello, R. Etienne-Cummings, and K. Boahen, "A biomorphic digital image sensor," IEEE Journal of Solid State Circuits, vol. 38, no. 2, pp. 281-294, 2003.

[16] G. Indiveri , E. Chicca, and R. Douglas, "A VLSI array of low-power spiking neurons and bistable synapses with spike-timing dependent plasticity," IEEE Trans. Neural Networks, vol. 17, no. 1, pp. 211-221, 2006.

[17] K. Nakada, T. Asai, and H. Hayashi, "Analog VLSI implementation of resonate-and-fire neuron," International Journal of Neural Systems, vol. 16, no. 6, pp. 445-456, 2006.

[18] J. Wijekoon and P. Dudek, "Compact silicon neuron circuit with spiking and bursting behaviour," Neural Networks, vol. 21, no. 2-3, pp. 524-534, 2008.

[19] A. van Schaik, C. Jin, A. McEwan, and T.J. Hamilton, "A log-domain implementation of the Izhikevich neuron model," in Proc. IEEE ISCAS, pp. 4253-4256, 2010.

[20] A. van Schaik, C. Jin, A. McEwan, T.J. Hamilton, S. Mihalas, and E. Niebur, "A log-domain implementation of the Mihalas-Niebur neuron model," in Proc. IEEE ISCAS, pp. 4249-4252, 2010.

[21] S. Millner, A. Grübl, J. Schemmel, K. Meier, and M.O. Schwartz, "A VLSI implementation of the adaptive exponential integrate-and-fire neuron model," Advances in Neural Information Processing Systems, vol. 23, pp. 1642-1650, 2010.

[22] N. Mizoguchi, Y. Nagamatsu, K. Aihara, and T. Kohno, "A two-variable silicon neuron circuit based on the Izhikevich model," Artificial Life and Robotics, vol. 16, no. 3, pp. 383-388, 2011.

[23] P. Livi and G. Indiveri, "A current-mode conductance-based silicon neuron for address-event neuromorphic systems," in Proc. IEEE ISCAS, pp. 2898-2901, 2009.

[24] H. Chen, S. Saïghi, L. Buhry, and S. Renaud, "Real-time simulation of biologically realistic stochastic neurons in VLSI," IEEE Trans. Neural Networks, vol. 21, no. 9, pp. 1511-1517, 2010.

[25] M. Simoni, G. Cymbalyuk, M. Sorensen, R. Calabrese, and S. DeWeerth, "A multiconductance silicon neuron with biologically matched dynamics," IEEE Trans. Biomedical Engineering, 
vol. 51, no. 2, pp. 342-354, 2004.

[26] G. Cymbalyuk, G. Patel, R. Calabrese, S. DeWeerth, and A. Cohen, "Modeling altemation to synchrony with inhibitory coupling: A neuromorphic VLSI approach," Neural Computation, vol. 12, pp. 2259-2278, 2000.

[27] E. Farquhar and P. Hasler, "A bio-physically inspired silicon neuron," IEEE Trans. Circuits and Systems I, vol. 52, no. 3, pp. 477-488, 2005.

[28] T. Kohno, J. Li, and K. Aihara, "Silicon neuronal networks towards brain-morphic computers," NOLTA, vol. 5, no. 3, pp. 379-390, 2014.

[29] T. Kohno and K. Aihara, "Reducing a fluctuation in burst firing of a square-wave burster silicon neuron model," PhysCon, Spain, September 2011.

[30] A. Basu and P. Hasler, "Nullcline-based design of a silicon neuron," IEEE Trans. Crcuits and Systems I, vol. 57, no. 11, pp. 2938-2947, 2010.

[31] J. Arthur and K. Boahen, "Silicon-neuron design: A dynamical systems approach," IEEE Trans. Circuits and Systems I, vol. 58, no. 5, pp. 1034-1043, 2010.

[32] J. Arthur and K. Boahen, "Synchrony in silicon: The gamma rhythm," IEEE Trans. Neural Networks, vol. 18, no. 6, pp. 1815-1825, 2007.

[33] P. Gao, B.V. Benjamin, and K. Boahen, "Dynamical system guided mapping of quantitative neuronal models onto neuromorphic hardware," IEEE Trans. Circuits and Systems, in press, 2012.

[34] A. Adamatzky, B.D.L. Costello, and T. Asai, Reaction-diffusion computers, Elsevier Science, 2005.

[35] S. Suenaga, Y. Hayakawa, and K. Nakajima, "Design of a neural network chip for the burst ID model with ability of burst firing," IEICE Trans. Fundamentals, vol. E90-A, pp. 715-723, 2007.

[36] A. Utagawa, T. Asai, and Y. Amemiya, "Stochastic resonance in simple analog circuits with a single operational amplifier having a double-well potential," NOLTA, vol. 2, no. 4, pp. 409-416, 2011.

[37] K. Nakada and T. Asai, Presented at IEICE CCS Technical Workshop, Tokyo, Japan, March, 2012.

[38] K. Nakada, K. Miura, and T. Asai, Presented at IEICE CAS Technical Workshop, Fukuoka, Japan, January, 2012.

[39] K. Nakada, S. Yakata, and T. Kimura, "Noise-induced synchronization in spin torque nano oscillators," Journal of Applied Physics, vol. 111, no. 7, 07C920, 2012.

[40] E.M. Izhikevich, Dynamical Systems in Neuroscience: the Geometry of Excitability and Bursting, The MIT press, 2007.

[41] Y. Kuramoto, Chemical oscillations, waves, and turbulence, Springer-Verlag, Berlin and New York, 1984.

[42] A.H. Cohen, P.J. Holmes, and R.H. Rand, "The nature of the coupling between segmental oscillators of the lamprey spinal generator for locomotion: A mathematical model," J. Mathematical Biology., vol. 13, pp. 345-369, 1982.

[43] J. Ladenbauer, M. Augustin, L. Shiau, and K. Obermayer, "Impact of adaptation currents on synchronization of coupled exponential integrate-and-fire neurons," PLoS Comput Biol., vol. 8, no. $4,2012$.

[44] K. Miura and M. Okada, "Globally coupled resonate-and-fire models," Progress of Theoretical Physics Supplement, vol. 161, pp. 255-259, 2006.

[45] K. Miura and K. Nakada, Presented at SCIS-ISIS, Kobe Japan, November 2012.

[46] K. Matsuzaka, T. Takashi, K. Nakada, and T. Morie, "Analog CMOS circuit implementation of a pulse-coupled phase oscillator system and observation of synchronization phenomena," NOLTA, vol. 3, no. 2, pp. 180-190, 2012.

[47] Y.D. Sato and K. Aihara, "Changes of firing rate induced by changes of phase response curve in bifurcation transitions," Neural Computation, vol. 26, no. 11, pp. 2395-2418, 2014. 